\title{
The Manchester Respiratory-related Sleep Symptoms scale for patients with COPD: development and validation
}

This article was published in the following Dove Press journal: International Journal of COPD

\section{Naimat Khan' \\ Jørgen Vestbo ${ }^{2}$ \\ Adam Garrow ${ }^{3}$ \\ Pradeep Karur ${ }^{4}$ \\ Umme Kolsum ${ }^{5}$ \\ Sarah Tyson ${ }^{6}$ \\ Dave Singh $^{7}$ \\ Janelle Yorke ${ }^{8}$}

'The Medicines Evaluation Unit, Wythenshawe Hospital, Manchester, UK; ${ }^{2}$ University of Manchester, Manchester University NHS

Foundation Trust, Manchester, UK; ${ }^{3}$ Division of Population Health, University of Manchester, Manchester, UK; ${ }^{4}$ Medicines Evaluation Unit, Manchester, UK; ${ }^{5}$ University of Manchester, Manchester, UK; ${ }^{6}$ University of Manchester, School of Health Sciences, Manchester Academic Health Sciences Centre, Manchester, UK; ${ }^{7}$ University of Manchester, The Medicines Evaluation Unit, Manchester, UK; ${ }^{8}$ University of Manchester, School of Health Sciences, Manchester, UK
Correspondence: Janelle Yorke School of Health Sciences, Faculty of Biology, Medicine and Health, University of Manchester, Room 5.320, Jean McFarlane University, Oxford Road, Manchester MI 3 9PL, UK

Tel +440 I6I 3067780

Email janelle.yorke@manchester.ac.uk
Background: In COPD disturbed sleep is related to exacerbation frequency, poor quality of life, and early mortality. We developed the Manchester Respiratory-related Sleep Symptoms scale (MaRSS) to assess sleep-time symptoms in COPD.

Methods: Focus groups including COPD and age-matched controls were used to develop an item-list, which was then administered to COPD patients and age-matched controls in a cross-sectional study. Hierarchical and Rasch analysis informed item selection and scale unidimensionality. Construct validity was examined using Pearson's correlation with the Sleep Problems Index, St George's Respiratory Questionnaire (SGRQ), and FACIT-Fatigue scale. MaRSS change scores from baseline (stable) to exacerbation were assessed in a separate substudy of COPD patients.

Results: Thirty-six COPD patients and nine age-matched controls produced an initial 26-item list. The cross-sectional study involved 203 COPD patients (male: 63\%, mean age 64.7 years) and 50 age-matched controls (male: $56 \%$, mean age 65.8 years). Eighteen items were removed to develop an eight-item unidimensional scale covering breathlessness, chest tightness, cough, sputum production, lack of sleep, and medication use. MaRSS scores significantly correlated with sleep problems, SGRQ Total, and FACIT-Fatigue $(r=0.58-0.62)$ and demonstrated a good fit to the Rasch model (chi-squared=29.2; $P=0.04$ ). In the substudy, MaRSS scores demonstrated a moderate effect size from baseline to exacerbation visit in 27 patients with 32 exacerbation episodes (Cohen's d=0.6).

Conclusion: The MaRSS is a reliable, valid, and clinically responsive measure of respiratoryrelated symptoms that disturb sleep. It is simple to use and score, making it suitable for research and clinical practice.

Keywords: COPD, sleep, dyspnea, cough, sputum, outcome measure

\section{Introduction}

It is well known that long-term interruption of sleeping patterns can be detrimental to physical, emotional, and social functioning; ${ }^{1}$ and that sleep disturbance is common in people with COPD. Disturbed sleep is associated with anxiety, depression, pain, and predictive of exacerbations, respiratory-related emergency hospital visits, and poor health outcomes in people for COPD. ${ }^{2}$ Despite the prevalence of sleep disorders in COPD, our recent systematic review highlighted a deficiency of validated measures for sleep problems in COPD. ${ }^{3}$ To date, sleep research in COPD has relied on generic patient-reported outcome measures (PROM) such as The Pittsburgh Sleep Quality Inde ${ }^{4}$ and the Epworth Sleepiness Scale ${ }^{5}$ but neither have been validated and are not specific for patients with COPD. ${ }^{3}$ 
Where generic sleep-related instruments have been used in COPD populations, estimates of prevalence and impact of sleep disturbance have been inconsistent. ${ }^{3}$ In the absence of published COPD validation data, it is difficult to assess the meaning of such inconsistencies. Similarly, since such instruments were not developed with COPD patients, their items do not include potentially important respiratory-symptom items, such as cough, dyspnea, and phlegm, which may interfere with sleep in patients with COPD. This is an important gap to address because medicines designed to ameliorate nighttime symptoms in COPD may lead to less interrupted sleep due to respiratory symptoms. An instrument that captures respiratory-specific symptoms during night-time sleep may provide useful effectiveness data for COPD medicines. Therefore, there is a need for a COPD-specific instrument to capture respiratory symptom-related sleep disturbance in COPD. ${ }^{3,6}$ The aim of this project was to develop and test the reliability and validate a new PROM to assess respiratorysymptoms-related sleep disturbance specifically in patients with COPD.

\section{Materials and methods}

This work was part of a PROM development and validation program and includes the development of the Manchester Early Morning Symptoms Index. ${ }^{7}$ Two separate studies were performed, both approved by the relevant ethics research committee (reference 12/NW/0608 and 10/H1003/108, respectively). Informed signed consent was obtained from all participants. For both studies, participants were recruited from a database of volunteers with COPD and controls without COPD held within the Medicines Evaluation Unit, a respiratory clinical trials unit in Manchester, UK, that recruits patients from both primary and secondary care. We collected COPD diagnosis, spirometry values, body mass index, comorbidities, and years from COPD.

COPD patients were aged 40 years or older, had a diagnosis according to GOLD criteria, ${ }^{8}$ and $\geq 10$ pack-year smoking history. Inclusion criteria for controls (Study 1 only) were aged 40 years or older and included current-, past-, and nonsmokers but with no history of significant respiratory disease. Controls were included to eliminate items reflecting sleep disturbance associated with age or other conditions and to provide a final PROM focused on respiratory-related symptoms that were COPD-specific.

Participants with a chest infection in the previous 3 months or a history of other respiratory conditions such as asthma, cystic fibrosis, and lung cancer were excluded. We also excluded any participant with insufficient English skills to give informed consent and people who regularly engaged in activities that could interrupt normal sleeping patterns (eg, night shift workers).

\section{Study I: PROM development and preliminary validation}

This was conducted in four stages: 1) item generation, 2) cognitive debriefing, 3) hierarchical item reduction and Rasch analysis, and 4) preliminary validity testing with other established measures. The process was designed to be compatible with international guidance. ${ }^{9}$ Further details regarding methods are available in the Supplementary materials.

\section{Stage I: Item generation}

Semistructured focus groups were arranged where participants and controls separately discussed their sleep experience and patterns. Focus groups were facilitated by one of the authors (JY), an experienced qualitative researcher, and consisted between seven and ten participants in each group. Topics included reasons for interrupted sleep, the need for medication, and the overall impact that interrupted sleep had on their lives. Focus group transcripts were analyzed by one of the authors (JY) and research assistant and presented to the research team and discussed where consensus was reached for pilot items.

\section{Stage 2: Cognitive debriefing}

A subgroup of COPD patients and controls participated in cognitive debriefing interviews based around the Questionnaire Appraisal System guidelines, ${ }^{10}$ to ensure that the generated items were unambiguous and accurately reflected COPD-specific respiratory-related night-time symptoms. Participants also considered recall options, scaling, layout acceptability, and questionnaire format. Participants read out each draft item and described its meaning as well as offering any alternate wording. Results from Stages 1 and 2 were used to produce a draft item list for the item reduction stage (Stage 3).

\section{Stage 3: Item reduction and Stage 4: psychometric testing}

These stages took place concurrently. In Stage 3, COPD patients and controls completed the draft item-list. Hierarchical item reduction techniques and Rasch analysis (detailed below) identified items with optimal measurement properties.

In Stage 4, consecutive responders from Stage 3 were mailed a second copy of the item list and a global rating of 
change questionnaire 7 days after completing the draft item list (detailed below). Participants returned the completed questionnaires within 1 week using the prepaid addressed envelope provided. Intraclass correlation coefficients (ICC) examined test-retest reliability for participants who indicated that their general health had remained "about the same" between visits. Construct validity was tested using correlations between the final-item set and relevant comparator measures that are detailed below.

\section{Data collection for Stages 3 and 4}

Demographic data and responses to the draft item list were collected during a study visit to the study unit. Medical history, current medications, and lung function test results collected within 6 months of the study visit were extracted from medical notes. The study questionnaires included the following:

1. The draft scale consisting of 26 respiratory-related sleep items (informed by the results of Stages 1 and 2), each scored on a 5-point scale (never, rarely, sometimes, often, and always).

2. The MOS-Sleep scale (Medical Outcomes Survey): a 12-item measure of sleep problems in patients with chronic illnesses. Individual items are scored on a four- or six-point scale ranging from "none of the time" to "most of the time" and aggregated to a range of scores from 0 to $100 .{ }^{11}$ It has been widely used as an outcome measure in other respiratory conditions. ${ }^{12,13}$

3. The FACIT-Fatigue scale: assesses the impact of fatigue on daily activities and function and has been validated for use in COPD. ${ }^{14,15}$ It is scored on a five-point scale with total scores ranging from 0 to 52; higher scores indicate worse fatigue.

4. The St George's Respiratory Questionnaire-COPD (SGRQ-C): measures the impact of COPD on overall health, daily life, and perceived well-being. ${ }^{16}$ A total score is calculated from three components: Symptoms, Activity, and Impacts on a scale from 0 to 100 .

5. The modified Medical Research Council Dyspnea Scale (mMRC): contains five statements regarding activity limitations associated with breathlessness. Scores range from 0 to 4 with higher scores indicating greater impairment. ${ }^{17}$

6. A Global Rating of Change: completed 7 days later along with the 32-item list. The change score assessed the stability of participants' health (much better; somewhat better; about the same; somewhat worse; much worse) since their first study visit in order to perform test-retest stability of the new item list.

\section{Ethical approval and consent to participate}

Two separate studies were performed, both approved by the relevant ethics research committee: National Research Ethics Committee for Greater Manchester East (references 12/NW/0608 and 10/H1003/108, respectively). Informed signed consent was obtained from all participants.

\section{Data analysis}

Study I: Stages I (item generation) and 2 (cognitive debriefing)

The focus group transcripts were coded and analyzed using a modified thematic analysis approach, ${ }^{18}$ to produce a provisional item list that highlighted participants' key sleep-related respiratory symptoms.

\section{Stage 3 and Stage 4: Hierarchical item reduction and}

\section{Rasch analysis}

Normally distributed data were analyzed using parametric statistics and presented as means and SDs. Non-normally distributed data are presented as medians and IQRs as appropriate. Categorical data were analyzed using chi-squared $\left(\chi^{2}\right)$ statistics. $P$-values $<0.05$ were considered statistically significant, unless stated otherwise. A Bonferroni adjustment was applied to adjust for multiple chi-square tests during the Rasch analysis. ${ }^{19}$ All statistical analyses were conducted using IBM SPSS Statistics for Windows (Version 20.0) or RUM 2030 (www.eumlab.com).

Items were removed if they demonstrated significant correlations with age (Pearson's $r$ ); gender differences in individual item responses (Student's $t$-test $P<0.05$ ); high item-item correlations $(P>0.8)$; or poor discrimination between COPD patients and controls (Student's $t$-test). ICC examined test-retest repeatability with values $\geq 0.7$ indicating acceptable repeatability. ${ }^{20}$ Construct validity was assessed by correlating (Pearson's $r$ ) scores from the final item-set with the other instruments.

Items surviving hierarchical reduction were examined using Rasch analysis to investigate how the remaining items contributed to the underlying construct, which was sleep symptoms. Individual item fit was tested using residual and chi-square fit statistics and items with poorest fit to the Rasch model were removed. ${ }^{21}$ The process continued until fit to the Rasch model was achieved, indicated by a Bonferroni adjusted person item trait chi-square statistic $(P>0.01)$ and a Person Separation Index $>0.7$, indicating good internal consistency.

Distribution of patient and item-threshold distribution maps was used to show the distribution of frequency 
of sleep symptoms and item frequency (locations) along a linear (logit) scale. Items that span the full range of person estimates located within \pm 2 logits indicate a welltargeted scale. ${ }^{21}$

Convergent validity was assessed using Pearson's correlation with the final MaRRS eight items and SGRQ (total and subscales), FACIT-Fatigue, and Sleep Index $(P<0.05)$.

\section{Study 2: MaRSS exacerbation-related change scores}

In study 2, data were extracted from an ongoing study - the MRC Cohort, Identifying Blood and Sputum Biomarkers of COPD Exacerbations (COPD MAP study 10/H1003/108). ${ }^{22}$ Participants were recruited if they had a confirmed diagnosis of mild to very severe COPD (Stages 1 to 4$){ }^{7}$ no other respiratory diagnosis, and their condition was classified as "stable"; that is, they had not experienced an exacerbation of their COPD during the preceding 6 weeks.
Participants who met the selection criteria and agreed to participate attended the unit for a baseline assessment, which included spirometry and a battery of questionnaires (only Manchester Respiratory-related Sleep Symptoms scale $[\mathrm{MaRSS}]$ and COPD Assessment Test [CAT] ${ }^{23}$ are reported here).

Following the baseline visit, patients contacted the study team when they experienced an exacerbation of their respiratory symptoms. This was recorded as E0, and the patients were invited to attend a post-exacerbation follow-up visit 2 weeks later (E2) and at 6 weeks (E6) and 3 months (3-month data not reported). Baseline correlations between MaRSS and CAT were assessed using Pearson's correlation. Response to clinical change was calculated between MaRRS scores at each exacerbation time-point including baseline, E0, E2, and E6, using Cohen's d effect size (Baseline to E0; Baseline to E2, and Baseline to E6).

Table I Participant characteristics for Stages I and 2

\begin{tabular}{|c|c|c|c|}
\hline Characteristics & $\begin{array}{l}\text { Control focus } \\
\text { groups }(n=12)\end{array}$ & $\begin{array}{l}\text { COPD focus } \\
\text { groups }(n=36)\end{array}$ & $\begin{array}{l}\text { COPD cognitive } \\
\text { debriefing }(n=9)\end{array}$ \\
\hline Male n (\%) & $6(50.0)$ & $18(50.0)$ & $3(33.3)$ \\
\hline Age mean (SD) & $66.0(7.2)$ & $68.4(5.2)$ & $66.4(6.2)$ \\
\hline BMI mean (SD) & $28.7(3.7)$ & $27.2(4.8)$ & $28.5(7.7)$ \\
\hline \multicolumn{4}{|l|}{ Comorbidities } \\
\hline Circulatory n (\%) & $6(50.0)$ & $24(66.6)$ & $6(66.7)$ \\
\hline Musculoskeletal n (\%) & $8(66.7)$ & $13(36.1)$ & $4(44.4)$ \\
\hline Endocrine $\mathrm{n}(\%)$ & $2(16.7)$ & $10(27.7)$ & $2(22.2)$ \\
\hline Digestive n (\%) & $0(0.0)$ & $13(36.1)$ & $4(44.4)$ \\
\hline Nervous n (\%) & $4(33.3)$ & $9(25.0)$ & $4(44.4)$ \\
\hline Urinary n (\%) & $0(0.0)$ & $7(19.4)$ & $0(0.0)$ \\
\hline Cancer n (\%) & $0(0.0)$ & $6(16.6)$ & $0(0.0)$ \\
\hline Reproductive n (\%) & $\mathrm{I}(8.3)$ & $3(8.3)$ & $I(11.1)$ \\
\hline Skin n (\%) & $0(0.0)$ & $2(5.5)$ & $0(0.0)$ \\
\hline \multicolumn{4}{|l|}{ Smoking exposure } \\
\hline Current smokers n (\%) & $\mathrm{I}(8.3)$ & II (30.6) & $4(44.4)$ \\
\hline Past smoker n (\%) & $7(58.3)$ & $25(69.4)$ & $5(55.6)$ \\
\hline Never smoked n (\%) & $4(33.3)$ & $0(0.0)$ & $0(0.0)$ \\
\hline Pack years n (\%) & $20.0(16.7)$ & $40.9(15.1)$ & $43.7(8.2)$ \\
\hline \multicolumn{4}{|l|}{ Respiratory function } \\
\hline COPD duration in years mean (SD) & $\mathrm{N} / \mathrm{A}$ & $7.7(4.5)$ & II.2 (4.I) \\
\hline $\mathrm{FEV}_{1} \%$ predicted mean (SD) & $\mathrm{N} / \mathrm{A}$ & $52.1(18.7)$ & $53.9(14.1)$ \\
\hline $\mathrm{FEV}_{\mathrm{I}} / \mathrm{FVC}(\%)(\mathrm{SD})$ & N/A & $47.7(12.3)$ & $46.9(19.2)$ \\
\hline GOLD Grade I n (\%) & N/A & $2(6.1)$ & \\
\hline GOLD Grade 2 n (\%) & N/A & $14(42.4)$ & $7(77.8)$ \\
\hline GOLD Grade 3 n (\%) & $\mathrm{N} / \mathrm{A}$ & $14(42.4)$ & $2(22.2)$ \\
\hline GOLD Grade 4 n (\%) & N/A & $3(9.1)$ & $0(0.0)$ \\
\hline GOLD Grade missing data & $\mathrm{N} / \mathrm{A}$ & 3 & \\
\hline
\end{tabular}

Abbreviations: BMI, body mass index; FEV \% predicted, forced expiratory volume in I second expressed as a percentage of the predicted normal range for the individual; FEV / IFVC, the proportion of forced expiratory volume in I second/forced vital capacity exhaled in the first second of expiration; N/A, not applicable. 


\section{Results}

\section{Study I}

Stage I and 2 item generation: focus groups and cognitive debriefing meetings

Forty-eight participants (36 COPD and 12 controls) participated in eight focus groups; six focus groups with COPD patients (range from five to seven participants) and two control group focus groups (six in each group). Nine COPD participants completed the cognitive debriefing sessions (Table 1).

Stage 1 generated 26 statements about sleep and/or night-time disturbance. Twelve related to respiratory symptoms (breathlessness, coughing, phlegm, and so on), four to sleeping position, four with medication use, and six with the consequences of a lack of sleep (eg, tiredness, affected mood - See Table S1). Six statements were removed at the end of Stage 1 due to similarity with other items. This process was informed through cognitive interviewing and confirmed during research meetings with COPD experts (JY, MK, JV, and DS). The remaining 20 statements were arranged and formatted as questionnaire items.

\section{Stage 2: Item reduction}

Two hundred three COPD participants and 50 controls completed the questionnaire pack (Table 2). The two groups were fairly evenly matched for age, gender, BMI, and smoking history. COPD patients had worse levels of fatigue and disrupted sleep than the controls.

Fourteen participants in the COPD group indicated that they were "never" affected by any of the sleep symptoms items and were removed from the analysis. No differences were found in gender, age, and GOLD grade in patients responding no to pilot items. Similarly three patients (1.4\%) failed to respond to all scale items and were removed leaving 186/203 (91.6\%) available for hierarchical item reduction.

\section{Stage 3: Hierarchical item reduction and Rasch analysis} Ten items were removed during the hierarchical reduction stage. Three general items about difficulty getting to sleep were removed because of poor discrimination between COPD and controls. A further item relating to being bad-tempered due to an inability to sleep was removed as it correlated highly with age. Five items with high item-item correlations were removed because of item redundancy. Two of these related to medication use at night, two concerned disturbed sleep from coughing, and one related to chest tightness. The final deleted item (getting into a good sleeping position) showed significant gender differences - female responders scored significantly higher than male responders.
Table 2 Participant characteristics for Stages 3 and 4

\begin{tabular}{|c|c|c|}
\hline Characteristics & $\begin{array}{l}\text { COPD } \\
(n=203)\end{array}$ & $\begin{array}{l}\text { Controls } \\
(n=50)\end{array}$ \\
\hline Male n (\%) & $128(63.1)$ & $28(56.0)$ \\
\hline Age (years) & $64.8(7.5)$ & $65.8(6.9)$ \\
\hline BMI (mean (SD)) & $27.6(5.2)$ & $27.6(4.5)$ \\
\hline Current smokers n (\%) & $66(32.5)$ & $16(32.0)$ \\
\hline Pack years median (IQR) & $4 \mid(27-57)$ & $26(17-36)$ \\
\hline COPD duration in years (SD) & $7.5(5.3)$ & \\
\hline \multicolumn{3}{|l|}{ MOS Sleep Scale mean (SD) } \\
\hline Sleep disturbance & $39.8(25.2)$ & $32.2(27.0)$ \\
\hline Short of breath/headache & $26.2(27.9)$ & $5.2(13.2)$ \\
\hline Sleep adequacy & $52.3(27.4)$ & $45.2(27.2)$ \\
\hline Sleep problem index II & $41.7(20.0)$ & $38.3(3.4)$ \\
\hline FACIT-Fatigue mean (SD) & $31.5(13.1)$ & $38.3(8.4)$ \\
\hline \multicolumn{3}{|l|}{ SGRQ-C mean (SD) } \\
\hline Total & $51.9(20.9)$ & \\
\hline Symptom & $59.6(19.5)$ & \\
\hline Activity & $66.2(27.7)$ & \\
\hline Impact & $40.1(22.8)$ & \\
\hline \multicolumn{3}{|l|}{ mMRC Dyspnea Scale n (\%) } \\
\hline 0 & $20(9.9)$ & \\
\hline I & $64(31.5)$ & \\
\hline 2 & 40 (19.7) & \\
\hline 3 & $56(27.6)$ & \\
\hline 4 & $23(11.3)$ & \\
\hline $\mathrm{FEV}, \%$ predicted (\%) & $56.6(18.6)$ & \\
\hline $\mathrm{FEV}_{1} / \mathrm{FVC}(\%)$ & $48.3(13.4)$ & \\
\hline \multicolumn{3}{|l|}{ GOLD (n=|76) n (\%) } \\
\hline Grade I & $28(15.9)$ & \\
\hline Grade 2 & $83(47.2)$ & \\
\hline Grade 3 & $50(28.4)$ & \\
\hline Grade 4 & $15(8.5)$ & \\
\hline
\end{tabular}

Abbreviations: FEV \% predicted, forced expiratory volume in I second expressed as a percentage of the predicted normal range for the individual; $\mathrm{FEV}_{1} / \mathrm{FVC}$, the proportion of forced expiratory volume in I second/forced vital capacity; mMRC modified Medical Research Council Dyspnea Scale; MOS, Medical Outcomes Survey; SGRQ-C, St George's Respiratory Questionnaire-COPD.

After removal of these ten items, a further eight COPD participants were found to "never" have been affected by any of the remaining items; no differences were found in gender, age, and GOLD grade in patients responding no to pilot items. Their data were removed leaving 178 complete data sets that were subjected to unidimensional modeling analysis. The ten items subjected to Rasch analysis did not initially fit the Rasch Model (total-item chi-square: 50.2; df: 20; $P=0.0002)$. Two nonfitting items were removed resulting in an eight-item scale - the MaRSS (Table 3 and Table S2). 
Table 3 Details of item removal during hierarchical $(n=203)$ and Rasch $(n=\mid 78)$ analysis

\begin{tabular}{|l|l|l|}
\hline Item & Reason for removal & $P$-value or correlation \\
\hline Trouble getting to sleep & Unable to discriminate between COPD and controls & $0.8^{*}$ \\
\hline Interrupted sleep & Unable to discriminate between COPD and controls & $0.18^{*}$ \\
\hline Difficulty sleeping because of worry & Unable to discriminate between COPD and controls & $0.09^{*}$ \\
\hline Bad tempered because unable to sleep & Correlation with age & $0.0 I^{*}$ \\
\hline Use of COPD medication to help with sleep & Item redundant (item-item correlation) & $>0.8^{* *}$ \\
\hline Night-time use of medication & Item redundant (item-item correlation) & $>0.8^{* *}$ \\
\hline Cough while lying down & Item redundant (item-item correlation) & $>0.8^{* *}$ \\
\hline Difficulty sleeping because of coughing & Item redundant (item-item correlation) & $>0.8^{* *}$ \\
\hline Difficulty sleeping because of chest symptoms & Item redundant (item-item correlation) & $>0.8^{* *}$ \\
\hline Problems with sleeping position & Significant gender difference & $0.004^{*}$ \\
\hline Difficulty sleeping because of breathlessness & Rasch analysis & $<0.0 I^{* *}$ \\
\hline Difficulty going to sleep while lying & Rasch analysis & $<0.0 I^{*}$ \\
\hline
\end{tabular}

Note: *denotes P-values; **denotes correlations.

The MaRSS is a Likert-type scale where individuals are asked to describe their experience of sleep-related respiratory symptoms in the past 7 days. Items are scored 0 if the symptom has "never" applied to them; 1, if it "rarely" applies; 2, "sometimes"; 3, "often"; or 4, "always" producing a possible range of scores from 0 to 32 . The final MaRSS demonstrated good overall fit to the unidimensional model (chi-square 29.2, $P=0.04$ ) and a good distribution of item scores (ranging from 1.712 logits: "difficulty falling asleep because of phlegm/sputum" 1.536 logits "woke up feeling tired") (Table S2).

\section{Stage 4: Psychometric assessment of the eight-item MaRSS}

The MaRSS demonstrated excellent test reliability (PSI $=0.85$ ). Of the 186 responses considered in Stage 2, 92 (49.4\%) completed a second copy of the draft PROM (mean response 7.5; SD 5.8 days), 78/92 (84.7\%) of whom reported that their health had not changed since completing the original questionnaires. The MaRSS showed good test-retest repeatability ( $\mathrm{n}=78)$ (ICC $=0.77,95 \%$ CI $0.66-0.85)$.

Concurrent validity: The MaRSS total score showed good correlations with the Sleep Problem Index $(r=0.58$, $P<0.001)$; FACIT-Fatigue $(r=-0.59, P<0.001)$; SGRQ-C total score $(r=0.62)$ and its three subscales; Symptoms $(r=0.58)$; Activity $(r=0.41)$; and Impact on daily life $(r=0.63)$ (all $P<0.01$ ).

Discriminant validity: There was a progressive increase in MaRSS scores with severity of breathlessness (mMRC) from 3.3 (SD 2.6) in Grade 0-13.5 (SD 6.1) in Grade 4, ANOVA F $(4,181)=11.69, P<0.001$ (Figure 1).

Table 4 shows the difference in mean item scores on the MaRSS between COPD patients and the controls. Mean item scores in the controls were all significantly lower than the COPD patients. Only two mean item scores (items 5 and 20) in the control group were greater than 0.4. Both of these were more general items relating to impaired activities and feelings of tiredness as a result of disrupted sleep.

\section{Study 2}

Twenty-seven patients with 32 exacerbations (five patients had two recorded exacerbations) were included in the analyses (mean age was 68 years \pm 7 and median $\mathrm{FEV}_{1}$ was $56.0 \%$, range $20.1 \%-101.0 \%$ ). There was a strong correlation between the MaRSS and CAT $(r=0.9, P<0.01)$. There were significant $(P=0.007)$ increases in MaRSS score from baseline to exacerbation visit ( $\mathrm{E} 0$, mean difference $=4.36$ points), representing a medium effect size (Cohen's d 0.64), compared to a small effect size for the CAT (Cohen's d 0.49).

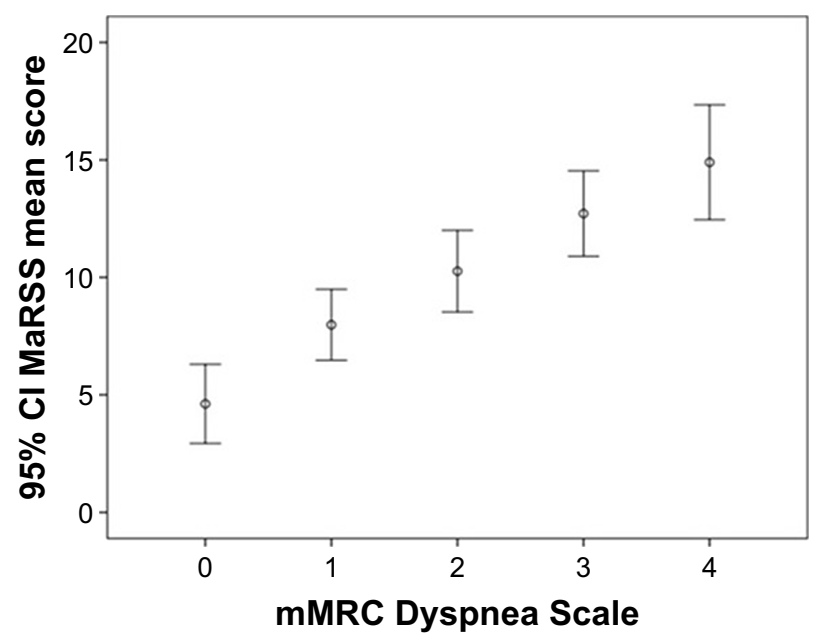

Figure I Association between mean MaRSS and mMRC scores (standard error and $\mathrm{Cl}$ ).

Abbreviations: MaRSS, Manchester Respiratory Sleep Symptoms; mMRC, modified Medical Research Council Dyspnea Scale. 
Table 4 Differences in mean item scores on the MaRSS between COPD patients and controls

\begin{tabular}{|l|l|l|l|}
\hline Final MaRSS items & $\begin{array}{l}\text { Mean score (SD) } \\
\text { COPD (n=186) }\end{array}$ & $\begin{array}{l}\text { Mean score (SD) } \\
\text { controls (n=50) }\end{array}$ & $\begin{array}{l}\boldsymbol{t} \text {-test } \\
\boldsymbol{P} \text {-value }\end{array}$ \\
\hline I woke up because of breathlessness & $\mathrm{I} .0 \mathrm{I}(\mathrm{I} .0)$ & $0.20(0.6)$ & $<0.00 \mathrm{I}$ \\
\hline Problems with sleep initiation due to phlegm & $0.99(0.99)$ & $0.28(0.7)$ & $<0.00 \mathrm{I}$ \\
\hline Sleep disruption due to phlegm & $\mathrm{I} .57(\mathrm{I} .2)$ & $0.84(0.9)$ & 0.002 \\
\hline Sleep disruption due to coughing & $\mathrm{I} .04(\mathrm{I} . \mathrm{I})$ & $0.08(0.4)$ & $<0.00 \mathrm{I}$ \\
\hline Sleep disruption due to chest symptoms & $\mathrm{I} .28(\mathrm{I} .1)$ & $0.32(0.6)$ & $<0.00 \mathrm{I}$ \\
\hline Tiredness & $0.9 \mathrm{I}(0.9)$ & $0.28(0.6)$ & $<0.00 \mathrm{I}$ \\
\hline Disruption of morning activities & $0.88(0.9)$ & $0.12(0.4)$ & $<0.00 \mathrm{I}$ \\
\hline Medication use & $2.02(\mathrm{I} .2)$ & $\mathrm{I} .36(\mathrm{I}) \mathrm{I})$ & $0.0 \mathrm{I}$ \\
\hline Total & $9.7(6.6)$ & $3.5(4.1)$ & $<0.00 \mathrm{I}$ \\
\hline
\end{tabular}

Abbreviation: MaRSS, Manchester Respiratory-related Sleep Symptoms scale.

Both MaRSS and CAT scores returned close to baseline scores at the post-exacerbation visit (E6) (Figure 2 and Table S3).

\section{Discussion}

We have described the development and validation of the MaRSS in line with the recommended good practice guidelines. ${ }^{9}$ A particular strength of this study was the inclusion of non-COPD control volunteers. This was to ensure that the final items on the MaRSS were specific to COPD respiratory-related symptoms that disturb night-time sleep. For focus group participants there were notable differences in musculoskeletal comorbidity between COPD and control participants (36\% and $66 \%$, respectively); however, no musculoskeletal-related sleep disturbances were expressed during focus group discussions. This unique feature of MaRSS will be of particular value when it is used to evaluate new medicines designed to ameliorate night-time respiratory-related symptoms such as cough, phlegm, and shortness of breath.

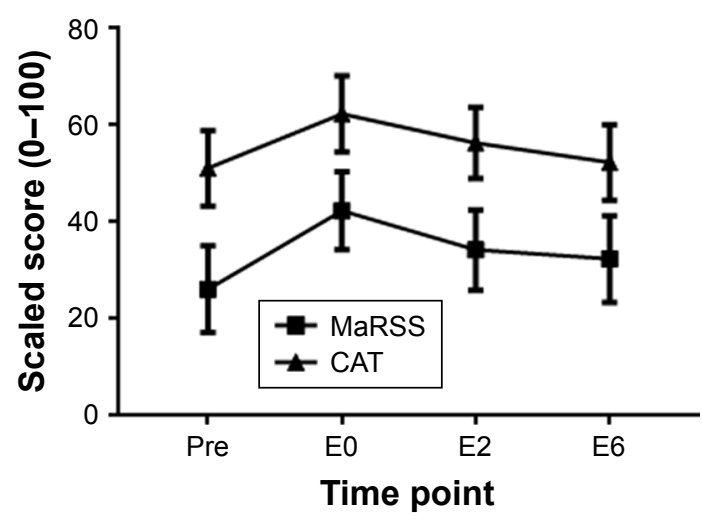

Figure 2 Comparison between MaRSS and CAT scores for each time point (scores are scaled 0-100) (standard error and $\mathrm{Cl}$ ).

Abbreviations: CAT, COPD Test; E0, day of exacerbation; E2, 2 weeks postexacerbation; E6, 6 weeks post-exacerbation; MaRSS, Manchester Respiratory Sleep Symptoms; Pre, baseline visit at study entry.
The final eight-item MaRSS is psychometrically robust. It is unidimensional (reflecting the underlying construct respiratory-related sleep problems), has good test reliability and test-retest repeatability, is suitable for patients with the full range of COPD severity, and is responsive to clinical change due to COPD exacerbation.

We found the MaRSS to be more sensitive to change than CAT (effect size 0.6 vs 0.49 , respectively). The reason for the observed difference requires further research; however, it may be related to the specific focus of the MaRSS items on respiratory-related sleep symptoms. MaRSS may be a useful marker of benefit from treatments designed to ameliorate respiratory symptoms at night and ultimately improve sleep in people with COPD.

The findings of the current study indicate that the MaRSS addresses the limitations of generic sleep measures highlighted in our previous review of sleep measures used in COPD. As it focuses on respiratory-related, rather than generic sleep symptoms, ${ }^{3}$ it will be more responsive to change due to respiratory-related sleep problems. The MaRSS opens up the possibility of being able to classify different phenotypes of patients based on disease-specific characteristics, which may respond to different treatment strategies and targeted sleep-based interventions. ${ }^{3}$

This study has some limitations. Participants were recruited using a single database of COPD patients living in Greater Manchester, so generalizability may be limited. Although our participants included patients with all grades of COPD severity, there were relatively few patients with very severe COPD (GOLD Grade 4: $n=15$ ). Cross-cultural validity of the MaRSS would also need to be established before it could be used in other countries with confidence. Although we demonstrated MaRSS responsiveness to clinical change, further research is needed to identify its minimal clinically important difference. 


\section{Conclusion}

We have outlined the development and preliminary validation of the MaRSS, a new PROM of sleep and night-time symptoms in COPD. The instrument has been shown to have a robust structure with good internal consistency, testretest reliability, validity, and responsiveness to clinical change. Further work is needed to confirm the usefulness of the MaRSS in intervention studies, determine Minimum Important Difference values, and establish cross-cultural validity.

\section{Availability of data and materials}

Available on request to the corresponding author.

\section{Acknowledgments}

This partnership received financial support from the Knowledge Transfer Partnerships programme (KTP). KTP is funded by the Technology Strategy Board along with the other government funding organizations (Grant number: KTP:8776). The project was also supported by the UK Medical Research Council.

\section{Disclosure}

The authors report no conflicts of interest in this work.

\section{References}

1. National Institutes of Health. Sleep, Sleep Disorders and Biological Rhythms; 2003. Available from: https://science.education.nih.gov/ supplements/nih_sleep_curr-supp.pdf. Accessed November 8, 2017.

2. Kessler R, Partridge MR, Miravitlles M, et al. Symptom variability in patients with severe COPD: a pan-European cross-sectional study. Eur Respir J. 2011;37(2):264-272.

3. Garrow AP, Yorke J, Khan N, Vestbo J, Singh D, Tyson S. Systematic literature review of patient-reported outcome measures used in assessment and measurement of sleep disorders in chronic obstructive pulmonary disease. Int J Chron Obstruct Pulmon Dis. 2015;10:293-307.

4. Buysse DJ, Reynolds CF, Monk TH, Berman SR, Kupfer DJ. The Pittsburgh Sleep Quality Index: a new instrument for psychiatric practice and research. Psychiatry Res. 1989;28(2):193-213.

5. Johns MW. A new method for measuring daytime sleepiness: the Epworth sleepiness scale. Sleep. 1991;14(6):540-545.

6. Agusti A, Hedner J, Marin JM, Barbé F, Cazzola M, Rennard S. Nighttime symptoms: a forgotten dimension of COPD. Eur Respir Rev. 2011; 20(121):183-194.

7. Garrow AP, Khan N, Tyson S, Vestbo J, Singh D, Yorke J. The development and first validation of the Manchester Early Morning Symptoms Index (MEMSI) for patients with COPD. Thorax. 2015;70(8): 757-763.
8. Vestbo J, Hurd SS, Agustí AG, et al. Global strategy for the diagnosis, management, and prevention of chronic obstructive pulmonary disease: GOLD executive summary. Am J Respir Crit Care Med. 2013;187(4): 347-365.

9. US Department of Health and Human Services Food and Drug Administration. Guidance for Industry Patient-Reported Outcome Measures: Use in Medical Product Development to Support Labeling Claims; 2009. Available from: http://www.fda.gov/downloads/Drugs/Guidances/ UCM193282.pdf. Accessed November 8, 2017.

10. Department of Health and Human Services Centres for Disease Control and Prevention. Evaluation Briefs Checklist to Evaluate the Quality of Questions; 2008. Available from: https://www.cdc.gov/healthyyouth/ evaluation/pdf/brief15.pdf. Accessed November 8, 2017.

11. Hays RD, Martin SA, Sesti AM, Spritzer KL. Psychometric properties of the Medical Outcomes Study Sleep measure. Sleep Med. 2005; 6(1):41-44.

12. Gotts ZM, Deary V, Newton J, van der Dussen D, de Roy P, Ellis JG. Are there sleep-specific phenotypes in patients with chronic fatigue syndrome? A cross-sectional polysomnography analysis. BMJ Open. 2013;3(6): $\mathrm{e} 002999$.

13. Murphy K, Nelson H, Parasuraman B, et al. The effect of budesonide and formoterol in one pressurized metered-dose inhaler on patient-reported outcomes in adults with mild-to-moderate persistent asthma. Curr Med Res Opin. 2008;24(3):879-894.

14. Yellen SB, Cella DF, Webster K, Blendowski C, Kaplan E. Measuring fatigue and other anemia-related symptoms with the Functional Assessment of Cancer Therapy (FACT) measurement system. J Pain Symptom Manage. 1997;13(2):63-74.

15. Al-Shair $\mathrm{K}$, Muellerova $\mathrm{H}$, Yorke J, et al. Examining fatigue in COPD: development, validity and reliability of a modified version of FACIT-F scale. Health Qual Life Outcomes. 2012;10:100.

16. Meguro M, Barley EA, Spencer S, Jones PW. Development and validation of an improved, COPD-specific version of the St. George respiratory questionnaire. Chest. 2007;132(2):456-463.

17. Medical Research Council. Modified Medical Research Council (mMRC) Dyspnea Scale. Available from: https://www.mrc.ac.uk/ research/facilities-and-resources-for-researchers/mrc-scales/mrcdyspnoea-scale-mrc-breathlessness-scale/. Accessed November 8, 2017.

18. Green J, Thorogood N. Qualitative Methods for Health Research. London: Sage Publications; 2004.

19. Cabin RJ, Mitchell RJ. To Bonferroni or not to Bonferroni: when and how are the questions. Bull Ecol Soc Am. 2000;81:246-248.

20. Bland JM, Altman DG. Statistical methods for assessing agreement between two methods of clinical measurement. Lancet. 1986; 1(8476):307-310.

21. Tennant A, Mckenna SP, Hagell P. Application of Rasch analysis in the development and application of quality of life instruments. Value Health. 2004;7(Supp1 1):S22-S26.

22. Kolsum U, Donaldson GC, Singh R, et al. Blood and sputum eosinophils in COPD; relationship with bacterial load. Respir Res. 2017; 18(1):88.

23. Jones PW, Harding G, Berry P, Wiklund I, Chen WH, Kline Leidy N. Development and first validation of the COPD Assessment Test. Eur Respir J. 2009;34(3):648-654. 


\section{Supplementary materials Study methods}

At each stage, participants were recruited from a database of volunteers with and without COPD (COPD $n \geq 800$ and nonCOPD $n>2,000$, respectively) at a respiratory clinical trials unit that works in collaboration with the University Hospital of South Manchester. Potential participants were contacted by telephone to ascertain their interest in taking part. If willing to participate an information pack and consent form were mailed to the patient and a study visit organized.

\section{Inclusion criteria}

Inclusion criteria were male and female patients aged 40 years or older diagnosed with COPD by a general practitioner or respiratory specialist using established criteria. These were $\mathrm{FEV}_{1}<80 \%$ of predicted normal, $\mathrm{FEV}_{1} /$ FVC $<0.70$, and symptoms consistent with COPD. Airflow limitation was graded according to post-bronchodilator $\mathrm{FEV}_{1}$ spirometry: GOLD was classified as $\mathrm{FEV}_{1} \geq 80 \%$ predicted: Grade 1 (mild); $50 \% \leq \mathrm{FEV}_{1}<80 \%$ predicted: Grade 2 (moderate); $30 \% \leq \mathrm{FEV}_{1}<50 \%$ predicted: Grade 3 (severe); $\mathrm{FEV}_{1}<30 \%$ predicted: Grade 4 (very severe).

Past and current smokers ( $\geq 10$ pack-year smoking history) were eligible to participate. Inclusion criteria were similar for the controls who may have had alternative long-term conditions, besides COPD. The reason for their inclusion was to eliminate sleep disturbance associated with age or other conditions so that, as far as possible, the final PROM focused on symptoms that were COPD-specific.

\section{Exclusion criteria}

Exclusion criteria included a chest infection in the previous 3 months; any other respiratory illness such as asthma, cystic fibrosis, and lung cancer; insufficient English skills to give informed consent and those regularly engaged in activities that could interrupt normal sleeping patterns (eg, night-shift workers).

\section{Global rating of change questionnaire}

Stage 4: To assess the test-retest reliability of the new measure. As part of the test-retest reliability of the MaRSS, participants were sent the item-list from Stage 3 and a global rating of change questionnaire. This assessed the stability of their health (much better; somewhat better; about the same; somewhat worse; much worse) since their first study visit. Participants returned the completed questionnaires within 1 week using the prepaid addressed envelope provided. Intraclass correlation coefficients examined test-retest reliability for participants who indicated their general health had remained "about the same" between visits.

Table SI Symptom areas produced from focus group discussions

\begin{tabular}{|l|l|l|}
\hline General symptom area & Number of items & Specific symptoms \\
\hline Respiratory & 12 & Breathlessness, chest tightness, wheezing, cough, phlegm \\
\hline Sleeping position & 4 & Getting comfortable, sitting, laying down \\
\hline Medication use & 4 & COPD medication to help with sleep and breathing \\
\hline Consequences of lack of sleep & 6 & Tiredness, mood affected \\
\hline
\end{tabular}

Table S2 Individual fit of the eight-item MaRSS to the Rasch unidimensional model

\begin{tabular}{|c|c|c|c|}
\hline Item & $\begin{array}{l}\text { Residual item } \\
\text { fit }( \pm \mathbf{2 . 5})\end{array}$ & $\begin{array}{l}\text { Chi-square } \\
\text { P-value* }\end{array}$ & $\begin{array}{l}\text { Logit (item } \\
\text { severity)*** }\end{array}$ \\
\hline Item 4: I had difficulty falling asleep because of phlegm (sputum) & 0.103 & 3.582 & 1.712 \\
\hline Item 17: I woke up because of tightness in my chest & -1.46 & 6.143 & 0.534 \\
\hline Item 16: I woke up because of phlegm (sputum) & -0.259 & 2.429 & 0.458 \\
\hline Item I: I woke up because of breathlessness & -2.133 & 6.288 & 0.232 \\
\hline $\begin{array}{l}\text { Item 9: During the night, I have needed to take puffs/inhalations of a } \\
\text { short-acting bronchodilator (eg, Ventolin/Bricanyl) }\end{array}$ & -0.7 & 0.948 & -0.147 \\
\hline Item I3: I woke up because of coughing & 1.208 & 0.778 & -0.371 \\
\hline Item 5: Lack of sleep interfered with my morning activities & 1.297 & 3.138 & -0.88 \\
\hline Item 20: I woke up feeling tired & 2.06 & 4.619 & -1.536 \\
\hline
\end{tabular}

Notes: *Chi-square $P$-value $>0.05$ meets unidimensional Rasch model assumptions. **The last column (logit) shows the mean level of severity for each item. Items are presented in descending order of severity (-ve logit indicates less severe and +ve indicates more severe).

Abbreviation: MaRSS, Manchester Respiratory-related Sleep Symptoms scale. 
Table S3 Exacerbation-related change scores for MaRSS and CAT questionnaires

\begin{tabular}{|c|c|c|c|c|}
\hline Measurement period & $\begin{array}{l}\text { MaRSS } \\
\text { (mean difference; } P \text {-value) }\end{array}$ & $\begin{array}{l}\text { Cohen's d } \\
\text { effect size }\end{array}$ & $\begin{array}{l}\text { CAT (mean difference; } \\
\text { P-value) }\end{array}$ & $\begin{array}{l}\text { Cohen's d } \\
\text { effect size }\end{array}$ \\
\hline Baseline - E0* & $4.36 ; P=0.007$ & 0.64 & $4.21 ; P=0.006$ & 0.49 \\
\hline Baseline - E2** & $0.87 ; P=0.44$ (NS) & 0.13 & $2.09 ; P=0.14$ & 0.24 \\
\hline Baseline - E6*** & $0.38 ; P=0.66(\mathrm{NS})$ & 0.06 & $0.71 ; P=0.54$ & 0.08 \\
\hline
\end{tabular}

Notes: *E0: Day of exacerbation; **E2: 2 weeks post exacerbation; ***E6: 6 weeks post exacerbation.

Abbreviations: CAT, COPD Assessment Test; MaRSS, Manchester Respiratory-related Sleep Symptoms scale; NS, no significant change.

\section{Publish your work in this journal}

The International Journal of COPD is an international, peer-reviewed journal of therapeutics and pharmacology focusing on concise rapid reporting of clinical studies and reviews in COPD. Special focus is given to the pathophysiological processes underlying the disease, intervention programs, patient focused education, and self management protocols.
This journal is indexed on PubMed Central, MedLine and CAS. The manuscript management system is completely online and includes a very quick and fair peer-review system, which is all easy to use. Visit http://www.dovepress.com/testimonials.php to read real quotes from published authors. 